\title{
Partitions for high-rise construction using phosphogypsum
}

\author{
Sergey Zolotukhin ${ }^{1}$, Olga Kukina ${ }^{1,}$, and Anatoly Abramenko ${ }^{1}$ \\ ${ }^{1}$ Voronezh State Technical University, Voronezh, Russia
}

\begin{abstract}
Gypsum blocks are usually used to make partitions in highrise construction. Reducing the cost of materials used in high-rise construction is an urgent task of modern material science. Phosphogypsum dihydrate, which has binding properties, is one of the large-tonnage waste. The authors have proved that, after years of storage in heaps, water-soluble phosphates, fluorides and other additives included in the structure of fresh phosphogypsum dissolved in water due to weathering (humidification-drying, freezing-thawing in a water-saturated state), and the calcium hydro- and dihydrogen phosphates ingressed in the lattice underwent complete hydrolysis and disintegration, thereby changing the physicochemical properties of phosphogypsum. The data obtained by the authors on the absence of water-soluble compounds of phosphorus, fluorine in stale phosphogypsum indicate its ecological purity and the possibility of application in housing construction. Having analyzed the data of modern methods of differential scanning calorimetry and scanning electron microscopy, the authors predicted and proved by the energy of dehydration of phosphogypsum dihydrate, lime, sandy loam, the possibility of obtaining non-flammable materials with sufficient strength for wall materials. Understanding the processes occurring in water films (the thickness of the water film, the pressure, the temperature and the $\mathrm{pH}$ of the aqueous extract of the mixture, the drying of the materials produced), made it possible to develop a technology for obtaining wall products from lime-sandy phosphogypsum material using typical silicate brick production equipment and vibropresses for key-cog blocks production.
\end{abstract}

\section{Introduction}

In mass construction of high-rise buildings, partitions made of gypsum blocks are widely used. This is due to the fact that the key-cog blocks are produced with a clear geometry, a smooth surface that does not need additional processing. The quality of the surface meets the requirements for finishing decoration - paperring, coating - which can greatly reduce the cost of finishing works. Inter-apartment partitions should have good sound insulation. The sound insulation of hollow gypsum key-cog blocks is $43.0 \mathrm{~dB}$. According to SniP 23-03-

\footnotetext{
* Corresponding author: lgkkn@rambler.ru
} 
2003, chapter "Noise isolation", soundproofing of interior partitions should be $41 \mathrm{~dB}$. A partition can be double or single. In the case of a double partition, there must be an air gap of $40 \mathrm{~mm}$. Electrical wiring and communications can be safely hiden into partitions made of gypsum blocks. In fact, this material is an alternative to traditional gypsum plasterboard. It also allows to build partitions much faster, since it does not require installation of a frame, but has large dimensions itself. In addition, the gypsum blocks are rather narrow, which saves space in the room.

Phosphogypsum dihydrate, which has binding properties, is one of the large-tonnage waste. The annual volume of its production in the world is 225 million tons per year, while no more than $5 \%$ is used, forming heaps, which accumulate over decades, occupying huge areas. In the literature there are no data on investigation of the properties of stale phosphogypsum.

Because of high content of gypsum dihydrate in phosphogypsum dihydrate, numerous studies on its processing have been aimed at obtaining gypsum using classical technologies: burning, cooking in gypsum-making boilers, autoclaving.

However, the use of phosphogypsum dihydrate as a basic material for processing by traditional technologies did not lead to a wide use of phosphogypsum due to high power inputs, which are 2-3 times higher than the ones for processing ordinary gypsum stone. Available developments in mechano-hydrochemical activation, pressing of materials based on gypsum binders enable the creation of energy-efficient and cheap materials using phosphogypsum dihydrate. The factors holding back their application include the instability of the properties of waste materials and the lack of knowledge in the theory of condensation hardening of these systems. The development of the technology using standard equipment for the production of wall materials will simplify the wider use of phosphogypsum in construction.

In view of the above, the objectives of this work are:

- to conduct research on the properties of stale phosphogypsum;

- to develop the theory of structure formation of composites;

- to obtain energy-efficient low-cost materials used in high-rise construction.

In the structure formation of composite building materials, the most important are the electrical interactions, which depend on the masses, the magnitude of the charges of their constituent particles, the distance between them, and the penetrability of the interaction medium. The dependences, proposed by B.V. Deryagin and L.D. Landau (Fig. 1), allow us to assume that these interactions are realized by alternating fields of attraction and repulsion formed around the particles that make up the structure of composite building materials.

A special place in the formation of the structure of composite building materials belongs to the water. Here and further, "water" means ions and colloidal particles of materials forming the composite dissolved in water. Water is a medium that sharply changes the dielectric constant of the medium, predetermining the interaction forces between the particles.

When the components of raw mixtures are mixed, water is adsorbed as films on hydrophilic particles. The maximum strength characteristics of the obtained materials are reached at thicknesses of water films $1 \cdot 10^{-7} \mathrm{~m}>\Delta>1 \cdot 10^{-9} \mathrm{~m}$.

At the temperature $65 \ldots 70^{\circ} \mathrm{C}$ and the change in the $\mathrm{pH}$ of water extracts (Fig. 2), water films change their thickness dramatically. At the same temperatures, the penetrating power of water sharply increases. 


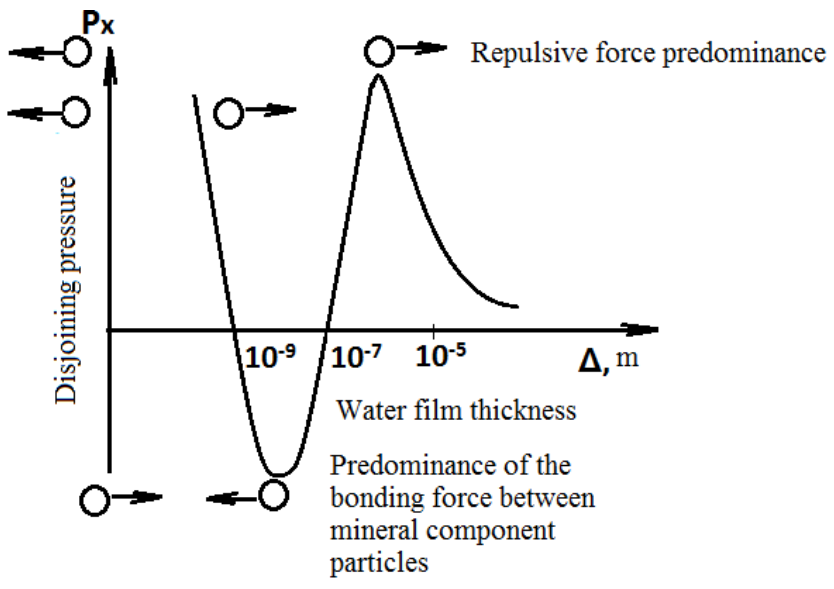

Fig. 1. The change in the disjoining pressure as a function of the thickness of the water film for hydrophilic particles

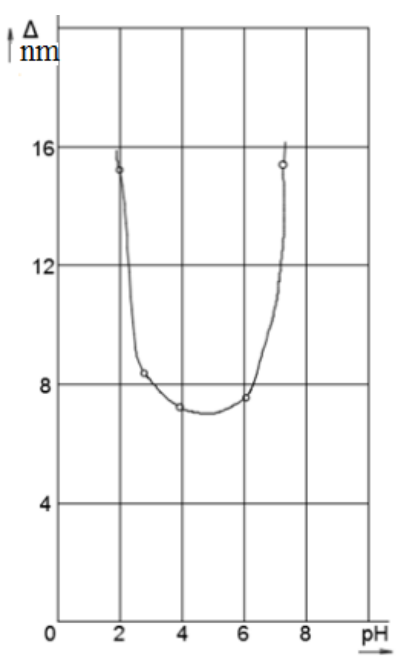

Fig. 2. Dependence of the thickness of the boundary layer on quartz particles on the $\mathrm{pH}$

\section{Materials, equipment and methods}

The following materials were used as raw mix components:

- construction quicklime (manufacturer: Rossosh, "Pridonkhimstroyizvest'", prepackaged: Voronezh, "Stroytorgservis");

- stale phosphogypsum dihydrate of the Uvarov Chemical Plant, liquidated in 2000 due to bankruptcy. The area of the heaps is $6.4 \mathrm{~km}^{2}$, the volume reaches about 35 million tons;

- sandy loam from the quarry of the Tambov region of the Uvarov district, located in the immediate vicinity of the phosphogypsum deposits.

The data of granulometric, microchemical, roentgen-phase, microscopic, and thermal analyzes are given in [1].

The physical and mechanical properties of the materials were determined in accordance with the requirements of GOST 23789-79, GOST 8736-93, GOST 5802-86, GOST 310.4.81, GOST 22688-77, GOST 125-79 on the universal electromechanical testing system Instron 5982 with the error in the load $\pm 0.5 \%$. X-ray phase analysis was performed on the automatic diffractometer PANalytical EMPYREAN. The differential scanning calorimetry (DSC) was performed on the synchronous thermal analysis device STA 449 F5 A-0082-M (NETSCH, Germany) with the NETSCH Proteus software with a built-in, reliable, monolithic weighing system and high-sensitivity DSC and DTA sensors in the modern device STA 449 F3 Jupiter $^{\circledR}$. Investigation of the microstructure of the materials was carried out using the scanning electron microscope JSM-6380LV. The images demonstrate the microstructure of the cleavages surface of the samples. The micron marker present in the images makes it possible to estimate the grain sizes and intergranular thicknesses of the water films.

Based on the results of the $\mathrm{pH}$ analysis of the water extracts of phosphogypsum dihydrate, data of microchemical, microscopic, X-ray phase and thermal analysis, it can be concluded that with age (from 1998 to 2017), the phosphogypsum changed its physicochemical parameters. Phosphoric acid, water-soluble phosphorus compounds, fluorine in the form of mechanical inclusions or compounds of the phosogypsum dihydrate crystalline lattice in the form of solid solutions have not been detected.

Analyzing the changes in the habit of phosphogypsum dihydrate, which are clearly visible 
when comparing the photomicrographs (Fig. 3, 4) of the stale phosphogypsum dihydrate, it can be concluded that over the years of storage in heaps, the water-soluble phosphates, fluorides and other impurities usually presented in the structure of fresh phosphogypsum dissolved in water due to weathering (humidification - drying, freezing - thawing in a saturated water state), and the calcium hydro- and dihydrogenphosphates ingressed in the crystal lattice underwent complete hydrolysis and disintegration under the action of the water molecules, thereby modifying the physicochemical properties of phosphogypsum.

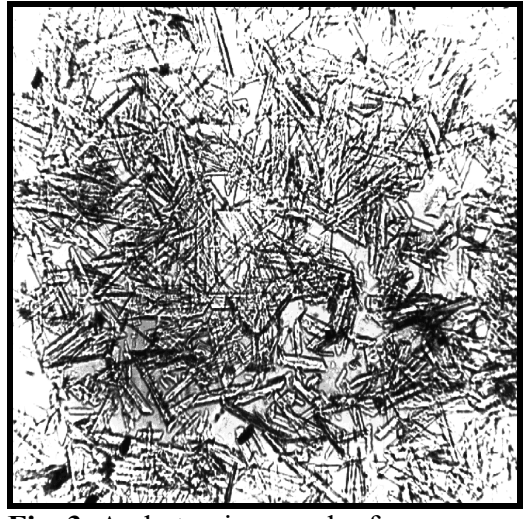

Fig. 3. A photomicrograph of phosphogypsum dihydrate at 300:1 magnification, made in 1998.

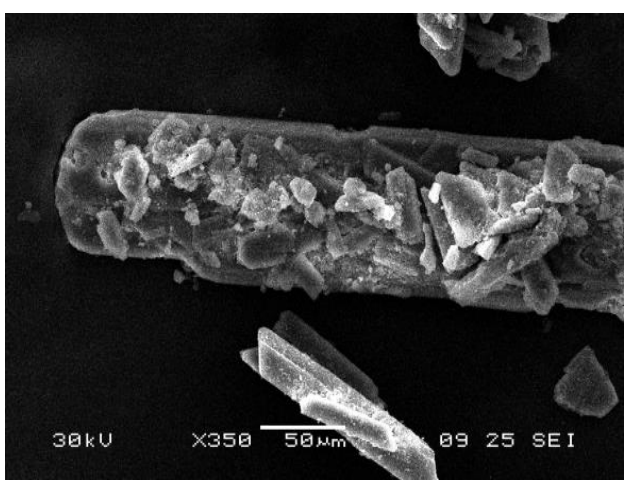

Fig. 4. A photomicrograph of the phosphogypsum dihydrate $(\times 350)$, made in 2017 .

The obtained data on the absence of water-soluble compounds of phosphorus, fluorine in stale phosphogypsum indicate its ecological purity and the possibility of application in housing construction, which is confirmed by the data of the "Center for Hygiene and Epidemiology in the Voronezh Region" [2].

The analysis of the results of the differential scanning calorimetry of materials used in the study showed the following:

- the energy of dehydration of the sandy loam of the Tambov region of the Uvarov district is $1.29 \mathrm{~J} / \mathrm{g}$, which indicates the absence of binding properties of this component of the lime-sandy phosphogypsum composite system (Fig. 5, 6);

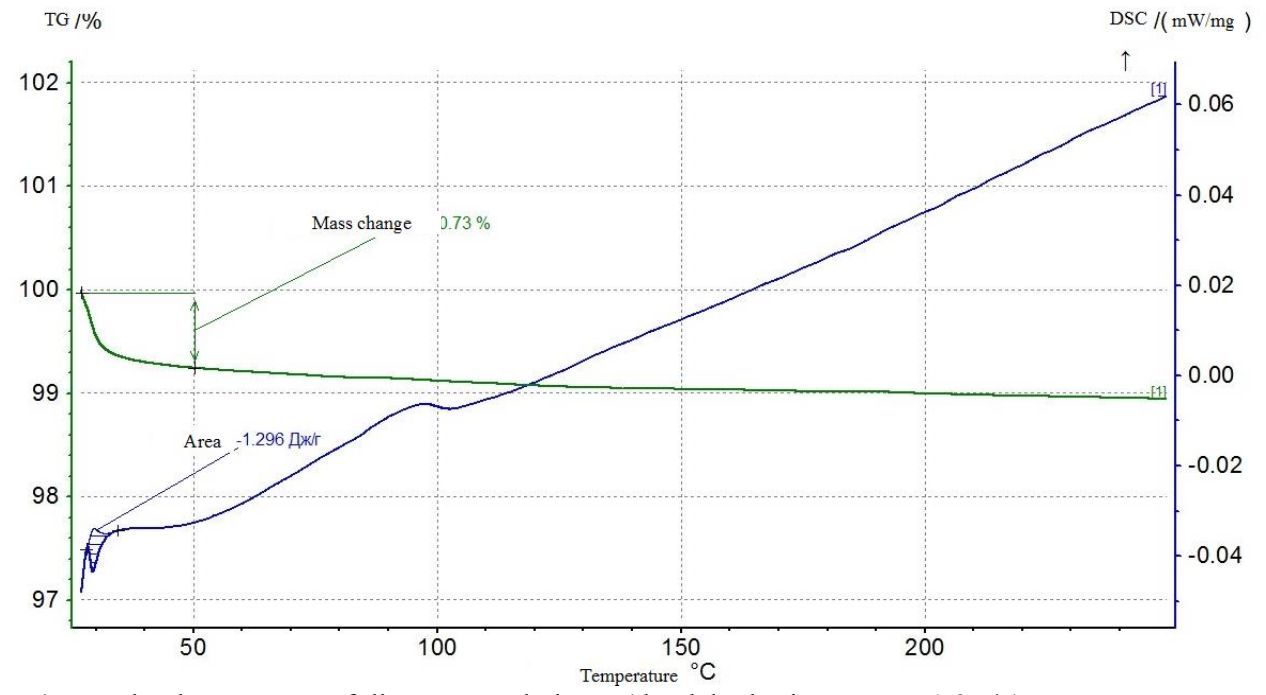

Fig. 5. The thermogram of disperse sandy loam (the dehydration energy $1.3 \mathrm{~J} / \mathrm{g}$ ) 
- the energy of dehydration in quicklime is more than $600 \mathrm{~J} / \mathrm{g}$. On the first day of hardening of hydrated lime, pressed at the pressure of $5 \mathrm{MPa}$, an endoeffect with the dehydration energy of $295.9 \mathrm{~J} / \mathrm{g}$ and the change in mass of $24.52 \%$ was observed. The 3 day of hardening of lime showed the endoeffect with the dehydration energy of $126.9 \mathrm{~J} / \mathrm{g}$ and the change in mass of $9.52 \%$. And by the 24 th day of hardening of hydrated lime, the endoeffect had the dehydration energy of $10.21 \mathrm{~J} / \mathrm{g}$ and the change in mass of $3.97 \%$. Such phase changes in lime confirm its binding properties only in the initial period of its hydration. However, in the case of the hydrated lime, the energy of dehydration decreases sharply with age, which indicates a decrease in binding properties in time due to the carbonization of its surface (Fig. 6-10). However, the high dispersion of lime allows to increase the number of crystallization contacts, which ultimately leads to an increase in the strength of the resulting materials;

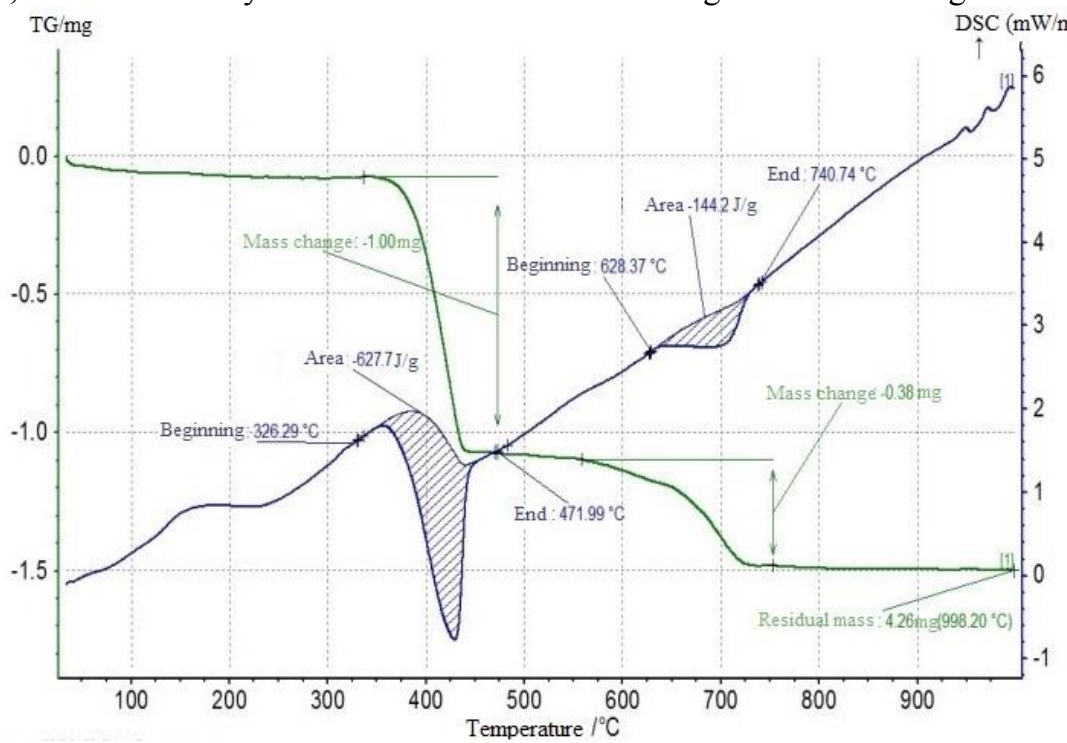

Fig. 6. The thermogram of a disperse sample of quicklime (the dehydration energy $772 \mathrm{~J} / \mathrm{g}$ )

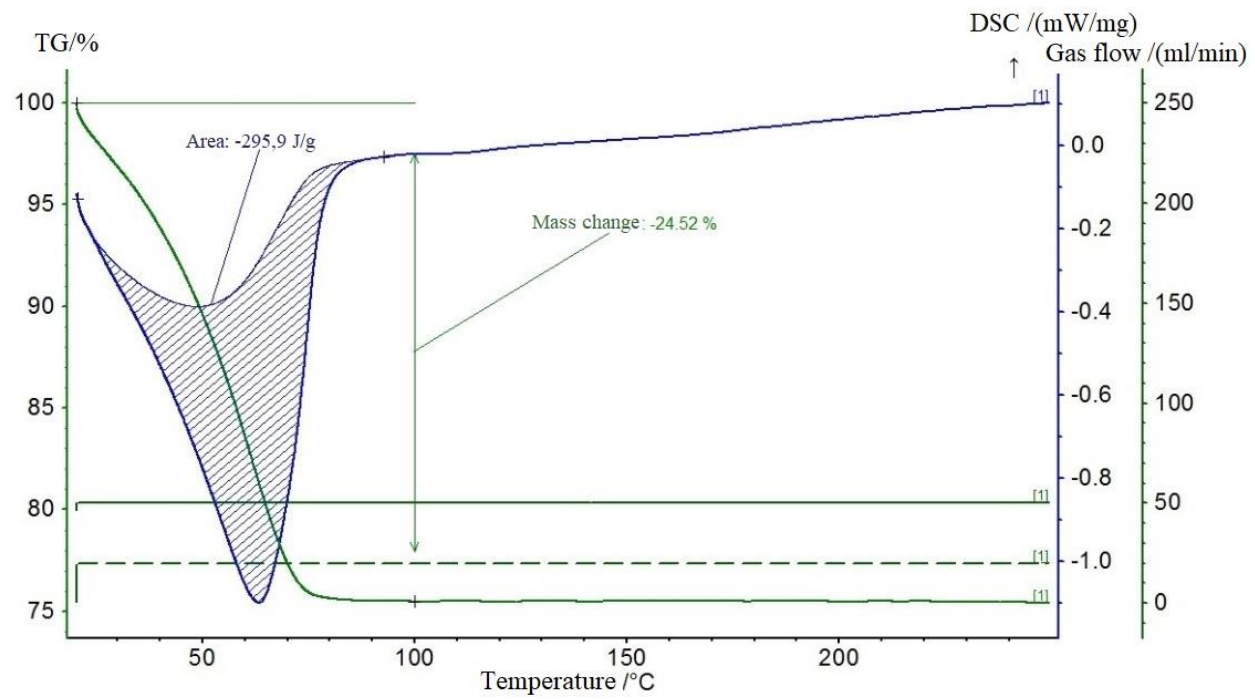

Fig. 7. The thermogram of a sample of hydrated lime pressed at $5 \mathrm{MPa}$ on the 1st day of hardening (the energy of dehydration $296 \mathrm{~J} / \mathrm{g}$ ) 


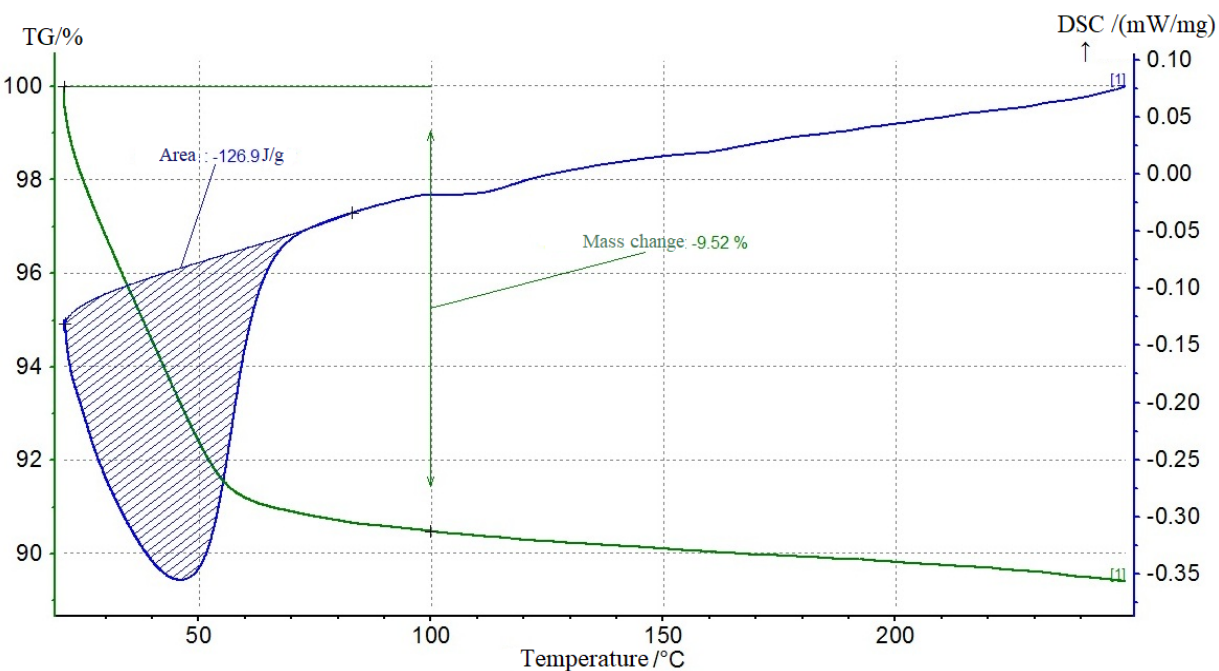

Fig. 8. The thermogram of lime pressed at $5 \mathrm{MPa}$ on the 3rd day of hardening

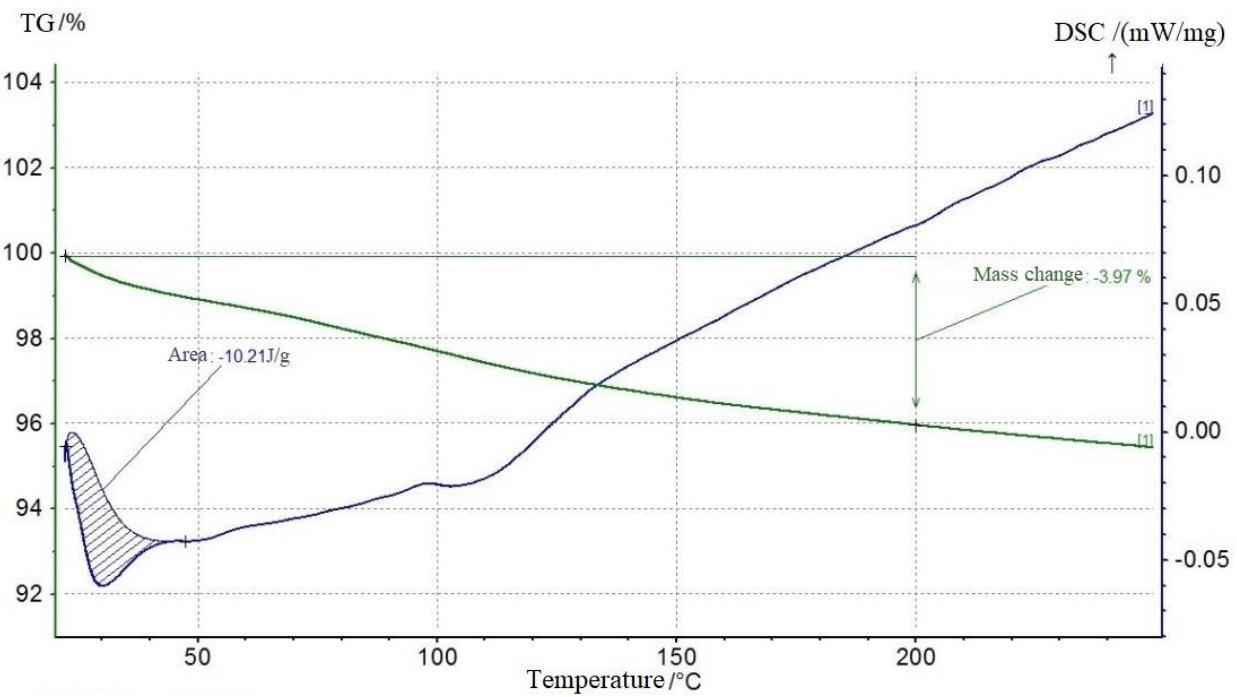

Fig. 9. The thermogram of a sample of hydrated lime pressed at $5 \mathrm{MPa}$ on the 24th day of hardening (the energy of dehydration $10 \mathrm{~J} / \mathrm{g}$ ) 
- the energy of dehydration of the stale phosphogypsum in 1998 (Fig. 11) and 2017 (Fig. 12-15), which show that over the years of storage in heaps, it has changed its binding properties. According to the thermographic studies of phosphogypsum dihydrate (Fig. 12), performed in 1998, the thermograms of phosphogypsum have a pronounced double endothermic effect of dehydration of the gypsum dihydrate, which is revealed in the interval $100-180^{\circ} \mathrm{C}$ [3]. By 2017 , the phosphogypsum has lost the pronounced double endothermic effect of dehydration of gypsum dihydrate. The onset of the first endoeffect of

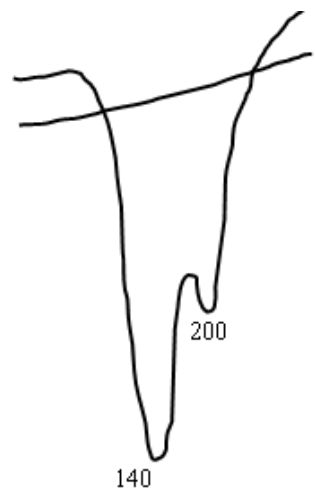

Fig. 10. The thermogram of phosphogypsum dihydrate (1998)

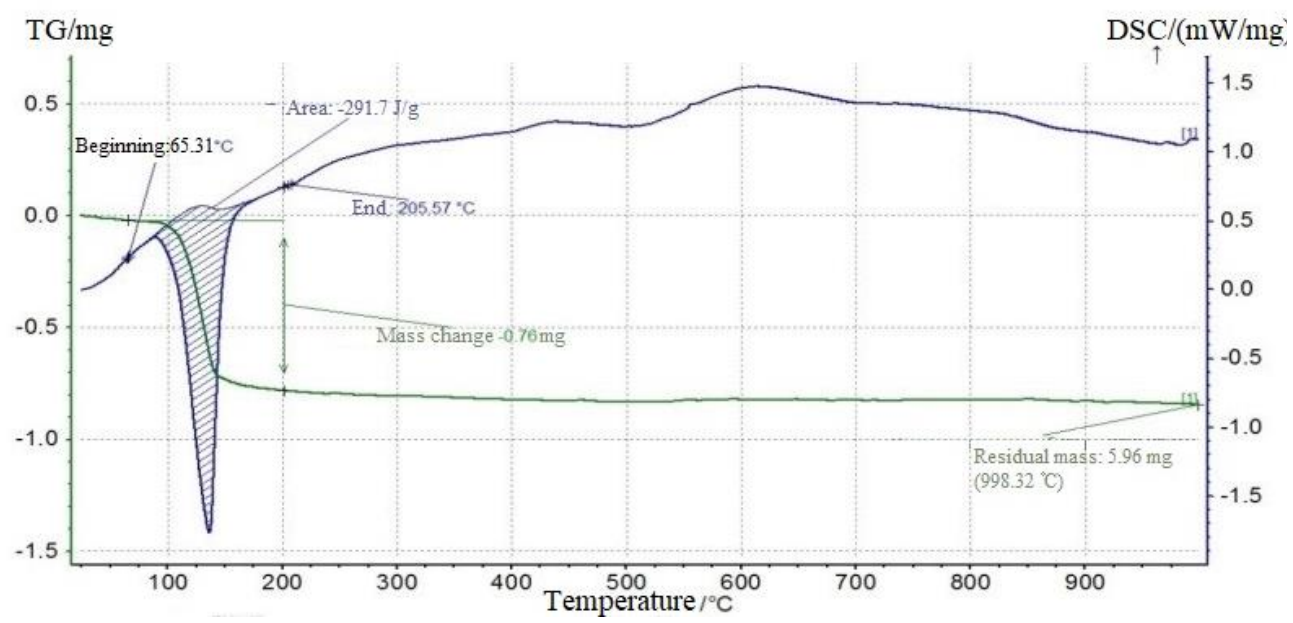

Fig. 11. The thermogram of phosphogypsum dihydrate (2017) (the energy of dehydration 291.7 $\mathrm{J} / \mathrm{g}$ )

The results of the differential-scanning calorimetry of pressed samples of the stale phosphogypsum dihydrate at the pressure of $5 \mathrm{MPa}$ are shown in Fig. 13 - 14. 


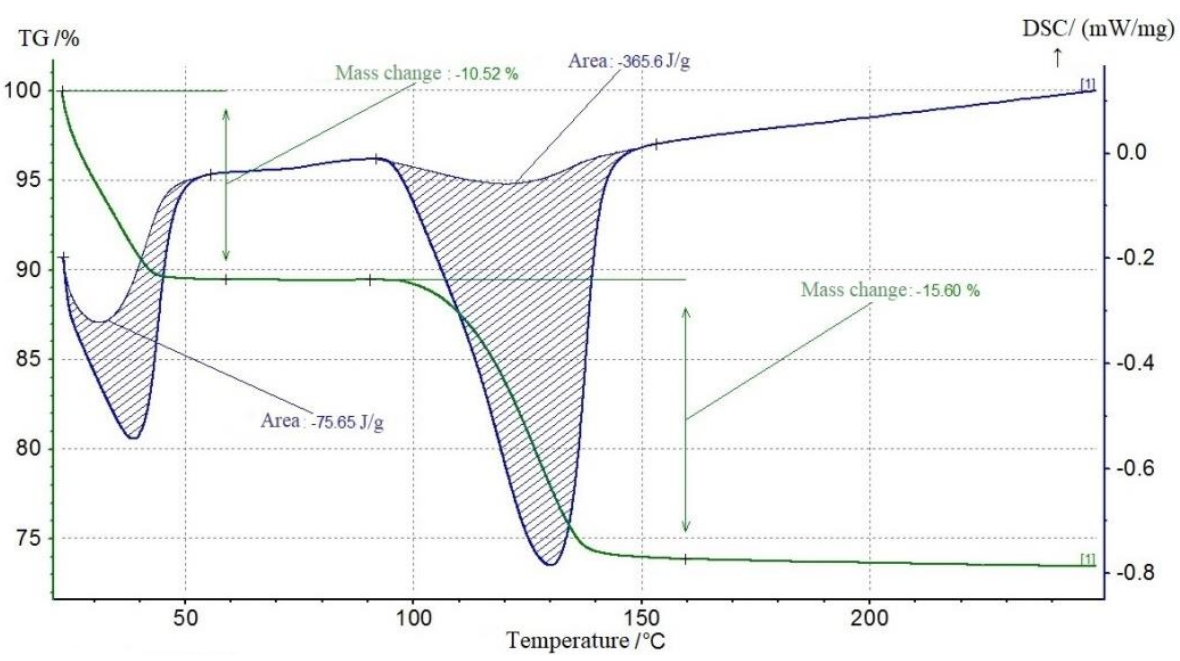

Fig. 12. The thermogram of phosphogypsum pressed at $5 \mathrm{MPa}$ on the 1st day of hardening (the dehydration energy $441 \mathrm{~J} / \mathrm{g}$ )

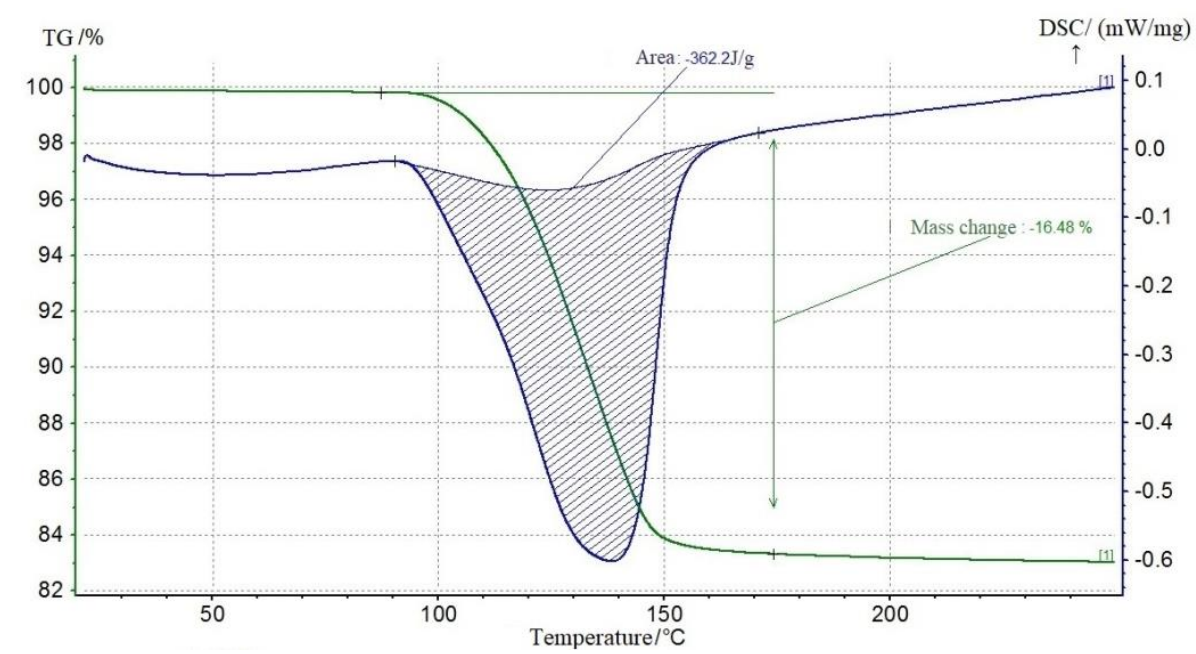

Fig. 13. The thermogram of phosphogypsum pressed at $5 \mathrm{MPa}$ on the 3rd day of hardening (the energy of dehydration $362 \mathrm{~J} / \mathrm{g}$ ) 


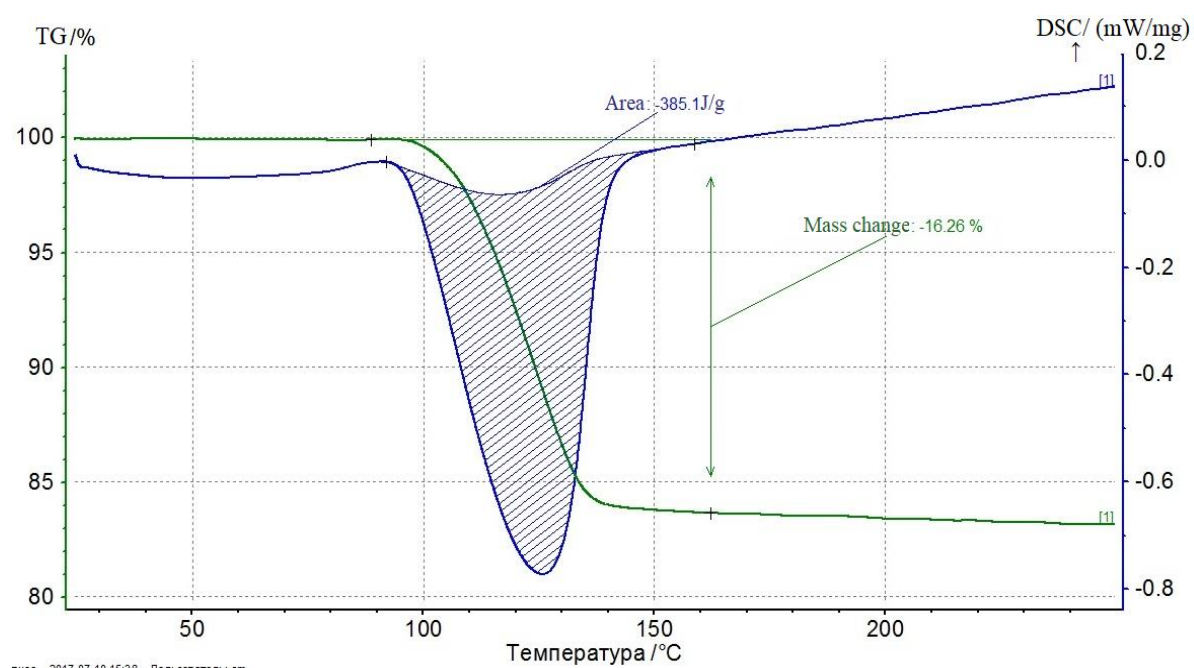

Fig. 14. The result of DSC of pressed phosphogypsum at $5 \mathrm{MPa}$ on the 24th day of hardening (dehydration energy $385 \mathrm{~J} / \mathrm{g}$ )

Previous studies [4] have shown that water films, self-organizing according to the usual technologies of preparation of mortars, stabilize at the thicknesses equal to $1 \cdot 10^{-7} \mathrm{~m}$, and the maximum strength parameters in the obtained materials can be reached at the thicknesses $1 \cdot 10^{-7} \mathrm{~m}>\Delta>1 \cdot 10^{-9} \mathrm{~m}$, so to reduce the thickness of the water films, it is necessary to bring the mineral components closer, which can be achieved by pressing or drying the resulting composites.

Understanding the contribution of each of the materials described above and the directional effect of the parameters of technological processes (thickness of the water film, pressure, temperature and $\mathrm{pH}$ of the aqueous extract of the mixture, drying of the produced materials) [5-21] allowed to develop a technology for obtaining wall products from limesandy phosphogypsum material on a typical equipment for the production of silicate bricks and vibropress plants for the production of key-cog blocks.

The raw meal for the production of building material included stale phosphogypsum dihydrate, hot water $\mathrm{t}=90-100^{\circ} \mathrm{C}$, dispersed sandy loam with the specific surface area of $4,000-4,500 \mathrm{~cm}^{2} / \mathrm{g}$ and quick lime, at $\mathrm{W} / \mathrm{S}=0.3-0.4$ [22].

The technical result showed the increase of strength of the material (Table 1), simplification and cheapening of the technology and improvement of the product quality.

Table 1. Dependence of the strength of lime-sandy phosphogypsum material on the pressure

\begin{tabular}{|c|c|c|}
\hline no & Pressure, MPa & Strength, MPa \\
\hline 1 & 2,5 & 6,74 \\
\hline 2 & 5 & 8,06 \\
\hline 3 & 10 & 11,50 \\
\hline 4 & 20 & 18,06 \\
\hline 5 & 25 & 19,72 \\
\hline 6 & 40 & 30,1 \\
\hline
\end{tabular}

Studies of the strength parameters of pressed lime-sandy phosphogypsum material have shown the possibility of direct production of building blocks with the strength of 5-20 MPa with a softening factor of 0.5-0.9. 
The cost of production of $1 \mathrm{~m}^{3}$ of wall gypsum materials, depending on the capacity of the equipment, varies from 900 to $1950 \mathrm{rubles} / \mathrm{m}^{3}$, which is 2 to 3 times cheaper than the analogues.

Thus, the developed technology of lime-sandy phosphogypsum material molding will allow to produce cheap effective gypsum products that can be used in production of structural materials for low- and medium-quality wall materials for internal works.

\section{Conclusions}

1. After years of storage in heaps, the water-soluble phosphates, fluorides and other additives included in the structure of fresh phosphogypsum dissolved in water due to weathering (humidification-drying, freezing-thawing in a water-saturated state), and the calcium hydro- and dihydrogen phosphates ingressed in the lattice underwent complete hydrolysis and disintegration, thereby changing the physicochemical properties of phosphogypsum.

2. Methods of differential scanning calorimetry according to the first endoeffects allow to predict binding properties of dispersed materials.

3. It has been registered that the binding properties of phosphogypsum dihydrate are changing with time. The thermograms show that the first endoeffects have the dehydration energy of more than $200 \mathrm{~J} / \mathrm{g}$ and do not disappear in time, which indicates that phosphogypsum shows binding properties constantly.

4. The contribution of highly dispersed sandy loam and hydrated lime materials to the strength of the resulting materials consists in the structuring of water films and the significant growth of crystallization contacts.

5. Water films, self-organizing according to the usual technologies of preparation of mortars, stabilize at thicknesses equal to $1 \cdot 10^{-7} \mathrm{~m}$, and the maximum strength parameters in the obtained materials can be reached at thicknesses $1 \cdot 10^{-7} \mathrm{~m}>\Delta>1 \cdot 10^{-9} \mathrm{~m}$, so to reduce the thickness of the water films, it is necessary to bring the mineral components closer, which can be achieved by pressing or drying the resulting composites.

6. Understanding the processes and the influence of each of the components of lime-sandy phosphogypsum material made it possible to develop the technology for the production of wall materials.

7. Studies of the strength parameters of pressed lime-sandy phosphogypsum material have shown the possibility of direct production of building blocks with the strength of 5-20 MPa with a softening factor of 0.5-0.9.

8. The cost of production of $1 \mathrm{~m}^{3}$ of wall gypsum materials, depending on the capacity of the equipment, varies from 900 to 1950 rubles $/ \mathrm{m}^{3}$, which is 2 to 3 times cheaper than the analogues.

9. The developed technology of molding of lime-sandy phosphogypsum material will allow to produce cheap effective gypsum products that can be used in production of structural materials for low- and medium-quality wall materials for internal works.

10. The results of this study allow to directly form the structures of composite building materials with any dispersed systems.

\section{References}

1. S.N. Zolotukhin, O.B. Kukina, A.A. Abramenko, E.A. Soloveva, E.A. Savenkova, IOP Conf. Ser.: Earth Environ. Sci. 90012088 (2017)

2. V.N. Semenov, PhD Thesis (2002)

3. G.D. Shmelev, PhD Thesis (1998) 
4. S.N. Zolotukhin, Dr. tech. sci. diss. (1990)

5. R.N. Mirsayev, V.V. Babkov, S.S.Yunusova, L.K. Kuznetsov, I.V. Nedoseko, A.I. Gabitov, Phosphogypsum waste of the chemical industry in the production of wall products (Moscow, 2004)

6. R.N. Mirsayev, V.V. Babkov, I.V. Nedoseko, S.S. Yunusova, I.I. Akhmadulina, U.Sh. Shayakhmetov, Const. mat. 6, 6-9, (2009)

7. I.V. Nedoseko, Full Dr. tech. sci. diss. (2002)

8. I.A. Gar'kina, A.M. Danilov, Ye.V. Korolev, Const. 3-4, 30-37 (2009)

9. E.V. Korolev, Nanotech. in constr.: Sci. Int. J. 1, 66-79 (2009)

10. A.N. Bormotov, I.A. Proshin, Ye.V. Korolev, Mathematical modeling and multicriteria synthesis of composite materials (Penza, 2011)

11. E.V. Korolev, V.A. Smirnov, Adv. Mat. Res. 746, 277-280 (2013)

12. A.F. Bur'yanov, Const. Mat. 8, 30-33 (2008)

13. G. Yakovlev, I. Polyanskikh, A. Gordina, G. Fedorova, A.B. Buryanov, 7th Sci.-Tech. Conf. on Mat. Prob. in Civ. Eng., MATBUD, 13-21 (2015)

14. Alaa M. Rashad, J. of Clean. Prod. 87, 717-725 (2015)

15. Liu Laibao, Zhang Yunsheng, Tan Kefeng, Adv. in Cem. Res. 27-10, 567-570 (2015)

16. Hua Sudong, Wang Kejin, Yao Xiao, Const. and Buil. Mat. 121, 290-299 (2016)

17. Yoon Seongjin, Mun Kyoungju, Hyung Wongil, J. of As. Arch. and Buil. Eng. 14-1, 189-195 (2015)

18. Shen Yan, Qian Jueshi, Huang Yongbo, Cem. \& Conc. Comp. 63, 67-75 (2015)

19. Shen Yan, Qian Jueshi, Adv. in Cem. Res. 27, 515-525 (2015)

20. B.Lamia, R.M. Jamel, Ch. Mohamed, Rom. J. of Mat. 47-1, 106-111 (2017)

21. Hua Sudong, Wang Kejin, Yao Xiao, Cem. \& Conc. Comp. 72, 299-308 (2016)

22. S.N. Zolotukhin, F. Ibragim, E.A. Savenkova, E.A. Soloveva, A.S. Lobosok, A.A. Abramenko, A.A. Drapalyuk, Yu.B. Potapov, Patent of the RF 2584018, (2016) 